Open J. Math. Sci., Vol. 1(2017), No. 1, pp. $34-43$

Website: https://pisrt.org/psr-press/journals/oms/

ISSN: 2523-0212 (Online) 2616-4906 (Print)

http://dx.doi.org/10.30538/oms2017.0004

\title{
QCD GHOST DARK ENERGY IN FRACTAL COSMOLOGY
}

\author{
INES G. SALAKO, FAIZA GULSHAN ${ }^{1}$
}

\begin{abstract}
We discuss the interacting QCD ghost dark energy with cold dark matter in the framework of Fractal cosmology. We investigate the cosmological parameters such as Hubble parameter, deceleration parameter and equation of state. We also discuss the physical significance of various cosmological planes like $\omega_{D}-\omega_{D}^{\prime}$ and state-finder. At the end, it is observed that all the results are compatible with observational data.

AMS Mathematics Subject Classification: 83C05.

Key words and phrases: fractal cosmology; HDE models; QCD ghost model; cosmological parameters; cosmological planes.
\end{abstract}

\section{Introduction}

The problem of accelerated expansion is a critical topic in cosmology since its discovery [1]. The main cause of accelerated expansion of the universe is a unknown force so-called dark energy (DE). To explain the nature of DE many cosmologists have proposed many models and theories. Many DE theories for dynamical DE scenario have been proposed to interpret the nature of accelerating universe. A number of DE models have been discussed in this context by many cosmologists. Cosmological constant $\Lambda(\Lambda C D M)[2]$ is the simplest candidate for DE (has a constant energy and pressure with constant equation of state). But this model has faced two major problems, cosmic coincidence and fine tuning [2].

In order to describe accelerated expansion phenomenon, two different approaches has been adopted. One is the proposal of various dynamical DE models such as family of chaplygin gas [3], holographic $[4,5]$, new agegraphic [6], polytropic

Received 20 Jun 2017. Revised 01 September 2017.

1 Corresponding Author

(C) 2017 Ines G. Salako, Faiza Gulshan. This is an open access article distributed under the Creative Commons Attribution License, which permits unrestricted use, distribution, and reproduction in any medium, provided the original work is properly cited. 
gas [7], pilgrim [8, 9, 10] $D E$ models etc. A second approach for understanding this strange component of the universe is modifying the standard theories of gravity, namely, General Relativity (GR) or Teleparallel Theory Equivalent to GR (TEGR). Several modified theories of gravity are $f(R), f(T)[11,12,13,14$, $15,16], f(R, \mathcal{T})[17,18], f(G)[19,20,21,22,23]$ (where $R$ is the curvature scalar, $T$ denotes the torsion scalar, $\mathcal{T}$ is the trace of the energy momentum tensor and $G$ is the invariant of Gauss-Bonnet defined as $G=R^{2}-4 R_{\mu \nu} R^{\mu \nu}+R_{\mu \nu \lambda \sigma} R^{\mu \nu \lambda \sigma}$ ). For clear review of DE models and modified theories of gravity, see the reference [24].

We arrange the paper as follow: Section $\mathbf{2}$ describes the basic equations of fractal cosmology. In section 3, we discuss the cosmological parameters (Hubble, Deceleration, EoS $)$ and planes $\left(\omega_{D}-\omega_{D}^{\prime}\right.$, state-finder). In the last section, we conclude our results.

\section{Fractal Cosmology}

According to Einstein gravity in a fractal space-time, the total action is $[25,26]$

$$
S=S_{G}+S_{m},
$$

where $S_{G}$ is the gravitational part of the action and can be defined as

$$
S_{G}=\frac{1}{16 \pi G} \int d \varrho(x) \sqrt{-g}\left(R-2 \Lambda-\omega \partial_{\mu} \nu \partial^{\mu} \nu\right)
$$

and $S_{m}$ is the matter part of the action is

$$
S_{m}=\int d \varrho(x) \sqrt{-g} \mathrm{七}_{m} .
$$

Where $g$ is the determinant of the metric (dimensionless) $g_{\mu \nu}, \Lambda$ is the cosmological constant and $R$ is the Ricci scalar, $\nu$ and $\omega$ are the fractional function and fractal parameter respectively, while the standard measure $d^{4} x$ is replaced with a Lebesgue-Stieltjes measure $d \varrho(x)$. The Friedmann equation in fractal universe can be obtained after variation of Eq.(1) with respect to the $g_{\mu \nu}$ as

$$
H^{2}+H \frac{\dot{\nu}}{\nu}-\frac{\omega}{6} \dot{\nu}^{2}=\frac{1}{3}\left(\rho_{d e}+\rho_{m}\right)+\frac{\Lambda}{3} .
$$

Here $H$ denotes the Hubble parameter $\left(H=\frac{\dot{a}}{a}\right), \rho_{c d m}$ and $\rho_{d e}$ are the energy densities due to CDM and DE and $p=p_{d e}$ is the pressure of DE. $k$ is the curvature constant with different values of $k=0,+1,-1$ described as a flat closed and open universe respectively. $\Lambda$ is the cosmological constant. The continuity equations in fractal universe are given by

$$
\begin{array}{r}
\dot{\rho}_{m}+\left(3 H+\frac{\dot{\nu}}{\nu}\right) \rho_{m}=Q, \\
\dot{\rho}_{d e}+\left(3 H+\frac{\dot{\nu}}{\nu}\right)\left(\rho_{d e}+p_{d e}\right)=-Q .
\end{array}
$$

Where $Q$ describes as the interaction term between $\mathrm{DE}$ and $\mathrm{CDM}$ with $Q=$ $3 b^{2} H \rho_{m}$ and $b^{2}$ is a coupling constant. 
By assuming a timelike fractal profile $\nu=a^{-\gamma}$ (where $\gamma$ is the constant), the Friedmann equation becomes

$$
H^{2}\left(1-\gamma-\frac{\gamma^{2} \omega a^{-2 \gamma}}{6}\right)=\frac{1}{3}\left(\rho_{d e}+\rho_{m}\right)
$$

and the continuity equations can be written as

$$
\begin{gathered}
\dot{\rho_{m}}=\left(3 b^{2}-3+\gamma\right) \rho_{m 0} a^{\left(3 b^{2}-3+\gamma\right)} H . \\
\dot{\rho}_{d e}+H(3-\gamma)\left(\rho_{d e}+p_{d e}\right)=-3 b^{2} H \rho_{m 0} a^{\left(3 b^{2}-3+\gamma\right)} .
\end{gathered}
$$

Where $\rho_{m 0}$ is the integrating constant.

\section{QCD Ghost Dark Energy}

Recent observations have been proved that Veneziane ghost of chromodynamics QCD is a good model and helps to solve the $\mathrm{U}(1)$ problem [27]. Veneziane ghost DE model contribute to the vacuum energy and proportional to $\Lambda_{Q C D}^{3} H$ (smallest $Q C D$ scale), where $H$ is the Hubble parameter and $\Lambda_{Q C D}$ is the QCD mass scale. GDE is defined as $[28,29,30,31,32] \rho_{d e}=\alpha H$. This model is discussed for many cosmological parameter theories and observational schemes. Later on, it has been discussed in the form $H+O\left(H^{2}\right)$ [33] of Veneziane ghost in QCD has enough vacuum energy by which the early evolution of the universe is explained (with the help of $H^{2}$ ) [34]. This model is called generalized ghost DE model (GGDE). Garcia-Salcedo has proposed a new version of GGDE called QCD ghost DE model which depends on the radius of trapping horizon. For flat universe, it is defined as

$$
\rho_{d e}=\frac{\alpha(1-\epsilon)}{\tilde{r}_{T}}
$$

where $\alpha$ is numerical constant, $\epsilon=\frac{\dot{\tilde{r}}_{T}}{2 H \tilde{r}_{T}}$ and $\tilde{r}_{T}=\frac{1}{H}$. Using these values in Eq.(10), we get

$$
\rho_{d e}=\alpha\left(1+\frac{\dot{H}}{2 H^{2}}\right) H
$$

3.1. Hubble Parameter. By using Eqs.(7) and (11), we get the Hubble parameter in the form

$$
\frac{\dot{H}}{H^{2}}=\frac{6}{\alpha}\left(H\left(1-\gamma-\frac{\gamma^{2} \omega a^{-2 \gamma}}{6}\right)-\frac{1}{3 H} \rho_{m 0} a^{\left(3 b^{2}-3+\gamma\right)}\right)-2 .
$$

We calculate the Hubble parameter numerically and its plot against the redshift parameter $(1+z)$ with the constant parametric values as shown in figure 1. We take $d^{2}=0.2,0.3,0.4, \alpha=0.5, \gamma=0.1$ and $b^{2}=0.2$. It can be observed from Figure 1 that Hubble parameter corresponds to future day observation of the universe. 


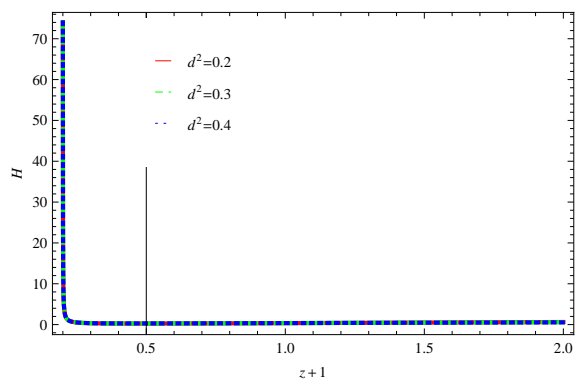

Figure 1. Plots of $H$ versus $1+z$ for QCD ghost DE model in fractal Cosmology.

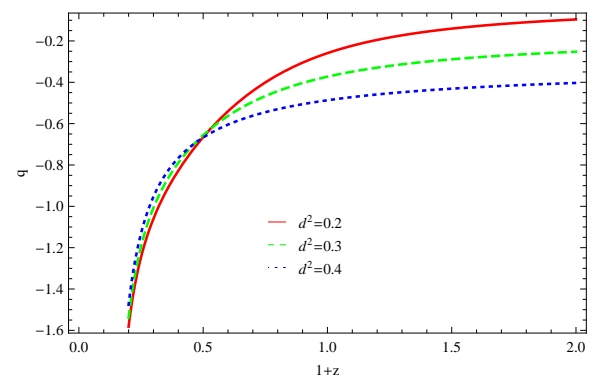

FIGURE 2. Plots of $q$ versus $1+z$ for QCD ghost DE model in fractal cosmology.

3.2. Deceleration Parameter. The deceleration parameter is denoted by $q$. This parameter tells us the transaction phase of the universe, either accelerating $(-1 \leq q<0)$ or decelerating $(q \geq 0)$. Its mathematical form is

$$
q=-\frac{\ddot{a} a}{\dot{a}^{2}}=-1-\frac{\dot{H}}{H^{2}} .
$$

After solving the Eqs. (13) and (12), the deceleration parameter is

$$
q=-1-\frac{6}{\alpha}\left(H\left(1-\gamma-\frac{\gamma^{2} \omega a^{-2 \gamma}}{6}\right)-\frac{1}{3 H} \rho_{m 0} a^{\left(3 b^{2}-3+\gamma\right)}\right)+2 .
$$

It is cleared from figure $\mathbf{2}$ that the deceleration parameter corresponds to acceleration expansion of the universe.

3.3. Equation of State Parameter. This parameter can be obtained by using Eqs.(9) and (12) as follows

$$
\omega_{d e}=-1-2\left(\alpha ( 3 - \gamma ) \left(2 H^{2}+\left(\frac { 6 } { \alpha } \left(H^{3}\left(1-\gamma-\frac{\gamma^{2} \omega a^{-2 \gamma}}{6}\right)-\frac{H}{3} \rho_{m 0}\right.\right.\right.\right.
$$




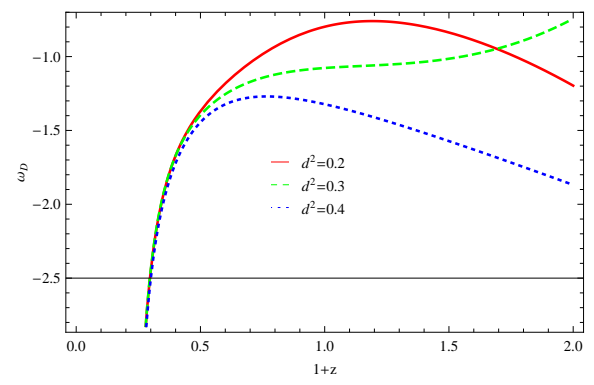

Figure 3. Plots of $\omega_{D}$ versus $1+z$ for QCD ghost DE model in fractal Cosmology.

$$
\begin{aligned}
& \left.\left.\left.\left.\times \quad a^{\left(3 b^{2}-3+\gamma\right)}\right)-2 H^{2}\right)\right)\right)^{-1}\left((3-\gamma) H \rho_{m_{0}} a^{\left(3 b^{2}-3+\gamma\right)}+\gamma^{3} \omega a^{-2 \gamma} H^{3}\right. \\
& +\left(\frac{6}{\alpha}\left(H^{3}\left(1-\gamma-\frac{\gamma^{2} \omega a^{-2 \gamma}}{6}\right)-\frac{H}{3} \rho_{m 0} a^{\left(3 b^{2}-3+\gamma\right)}\right)-2 H^{2}\right)(9 H \\
& \left.\times\left(1-\gamma-\frac{\gamma^{2} \omega a^{-2 \gamma}}{6}\right)-\rho_{m 0} a^{\left(3 b^{2}-3+\gamma\right)}\right)-\frac{\alpha}{2}\left(\frac { 6 } { \alpha } \left(H ^ { 3 } \left(1-\gamma-\frac{1}{6}\right.\right.\right. \\
& \left.\left.\left.\left.\times \quad \gamma^{2} \omega a^{-2 \gamma}\right)-\frac{H}{3} \rho_{m 0} a^{\left(3 b^{2}-3+\gamma\right)}\right)-2 H^{2}\right)^{2}\right) .
\end{aligned}
$$

The plot of EoS versus redshift parameter is shown in Figure 3. The EoS parameter behaves quintom-like nature for the interacting case $d^{2}=0.2$. For $d^{2}=0.3$, EoS parameter starts from phantom region and goes towards quintessence region of the universe. However, it remains in the phantom region for $d^{2}=0.4$.

3.4. $\omega_{D}-\omega_{D}^{\prime}$ plane. The $\omega_{D}-\omega_{D}^{\prime}$ plane characterize thawing as well as the freezing region of universe, i.e., when $\omega_{D}<0$ and $\omega_{D}^{\prime}>0$ then the plane corresponding to thawing region. But if both $\omega_{D}$ and $\omega_{D}^{\prime}$ are negative, then this plane provides freezing region. Caldwell and Linder [35] discover this method. By taking derivative of Eq.(15), we obtain

$$
\begin{aligned}
\omega_{D}^{\prime} & =-\frac{2}{H}\left(6(3-\gamma)\left(H^{3}\left(1-\gamma-\frac{1}{6} \gamma^{2} \omega a^{-2 \gamma}\right)-\frac{H}{3} \rho_{m 0} a^{\left(3 b^{2}-3+\gamma\right)}\right)\right)^{-1}((3 \\
& -\gamma)\left(3 b^{2}-3+\gamma\right) \rho_{m 0} a^{\left(3 b^{2}-3+\gamma\right)} H^{2}-2 \gamma^{4} \omega a^{-2 \gamma} H^{4}+\left(\frac { 6 } { \alpha } \left(H^{3}(1-\gamma\right.\right. \\
& \left.\left.\left.-\frac{\gamma^{2} \omega a^{-2 \gamma}}{6}\right)-\frac{H}{3} \rho_{m 0} a^{\left(3 b^{2}-3+\gamma\right)}\right)-2 H^{2}\right)\left((3-\gamma) a^{\left(3 b^{2}-3+\gamma\right)} \rho_{m 0}+3\right. \\
& \times \gamma^{3} \omega a^{-2 \gamma} H^{2}+\left(\frac{6}{\alpha}\left(H^{3}\left(1-\gamma-\frac{\gamma^{2} \omega a^{-2 \gamma}}{6}\right)-\frac{H}{3} a^{\left(3 b^{2}-3+\gamma\right)} \rho_{m o}\right)-2\right.
\end{aligned}
$$




$$
\begin{aligned}
& \left.\times \quad H^{2}\right)\left(9\left(1-\gamma-\frac{1}{6} \gamma^{2} \omega a^{-2 \gamma}\right)+\frac{1}{H^{2}} \rho_{m 0} a^{\left(3 b^{2}-3+\gamma\right)}\right)+3 \gamma^{3} \omega a^{-2 \gamma} H^{2} \\
& -\left(3 b^{2}-3+\gamma\right) \rho_{m 0} a^{\left(3 b^{2}-3+\gamma\right)}+\frac{\alpha}{H^{3}}\left(\frac { 6 } { \alpha } \left(H^{3}\left(1-\gamma-\frac{1}{6} \gamma^{2} \omega a^{-2 \gamma}\right)-\frac{1}{3}\right.\right. \\
& \left.\left.\times \quad \rho_{m 0} a^{\left(3 b^{2}-3+\gamma\right)} H\right)-2 H^{2}\right)^{2}-\frac{\alpha}{H^{2}}\left(\left(\frac{18}{\alpha} H^{2}\left(1-\gamma-\frac{1}{6} \gamma^{2} \omega a^{-2 \gamma}\right)-\frac{2}{\alpha}\right.\right. \\
& \left.\times \quad \rho_{m 0} a^{\left(3 b^{2}-3+\gamma\right)}-2 H\right)\left(\frac { 6 } { \alpha } \left(H^{3}\left(1-\gamma-\frac{1}{6} \gamma^{2} \omega a^{-2 \gamma}\right)-\frac{H}{3} a^{\left(3 b^{2}-3+\gamma\right)}\right.\right. \\
& \left.\left.\left.\times \quad \rho_{m 0}\right)-2 H^{2}\right)+\frac{2}{\alpha} \gamma^{3} \omega a^{-2 \gamma} H^{4}-\frac{2}{\alpha}\left(3 b^{2}-3+\gamma\right) a^{\left(3 b^{2}-3+\gamma\right)} \rho_{m 0} H^{2}\right) \\
& \times)+\left(( \frac { 1 8 } { \alpha } H ^ { 2 } ( 1 - \gamma - \frac { 1 } { 6 } \gamma ^ { 2 } \omega a ^ { - 2 \gamma } ) - \frac { 2 } { \alpha } \rho _ { m 0 } a ^ { ( 3 b ^ { 2 } - 3 + \gamma ) } - 2 H ) \left(\frac{6}{\alpha}(\right.\right. \\
& \left.\left.\times \quad H^{3}\left(1-\gamma-\frac{\gamma^{2} \omega a^{-2 \gamma}}{6}\right)-\frac{H}{3} \rho_{m 0} a^{\left(3 b^{2}-3+\gamma\right)}\right)-2 H^{2}\right)+\frac{2}{\alpha} \gamma^{3} a^{-2 \gamma} H^{4} \\
& \left.\times \quad \omega-\frac{2}{\alpha}\left(3 b^{2}-3+\gamma\right) \rho_{m 0} a^{\left(3 b^{2}-3+\gamma\right)} H^{2}\right)\left(9 H\left(1-\gamma-\frac{1}{6} \gamma^{2} a^{-2 \gamma} \omega\right)-\right. \\
& \left.\left.\times \quad \rho_{m 0} a^{\left(3 b^{2}-3+\gamma\right)}\right)\right)+\frac{2}{H}\left(\frac { 3 6 } { \alpha } ( 3 - \gamma ) \left(H^{3}\left(1-\gamma-\frac{1}{6} \gamma^{2} \omega a^{-2 \gamma}\right)-\frac{H}{3}\right.\right. \\
& \left.\left.\times \quad \rho_{m 0} a^{\left(3 b^{2}-3+\gamma\right)}\right)^{2}\right)^{-1}\left((3-\gamma) \rho_{m 0} a^{\left(3 b^{2}-3+\gamma\right)} H+\left(9 H \left(1-\gamma-\frac{1}{6} \gamma^{2}\right.\right.\right. \\
& \left.\left.\times \quad \omega a^{-2 \gamma}\right)-\frac{1}{H} \rho_{m 0} a^{\left(3 b^{2}-3+\gamma\right)}\right)\left(\frac { 6 } { \alpha } \left(H^{3}\left(1-\gamma-\frac{\gamma^{2} \omega a^{-2 \gamma}}{6}\right)-\frac{H}{3} \rho_{m 0}\right.\right. \\
& \left.\left.\times \quad a^{\left(3 b^{2}-3+\gamma\right)}\right)-2 H^{2}\right)+\gamma^{3} \omega a^{-2 \gamma} H^{3}-\frac{\alpha}{2 H^{2}}\left(\frac { 6 } { \alpha } \left(H ^ { 3 } \left(1-\gamma-\frac{1}{6} \gamma^{2} a^{-2 \gamma}\right.\right.\right. \\
& \left.\left.\left.\times \omega)-\frac{H}{3} \rho_{m 0} a^{\left(3 b^{2}-3+\gamma\right)}\right)-2 H^{2}\right)^{2}\right)\left(4 H \left(\frac { 6 } { \alpha } \left(H^{3}\left(1-\gamma-\frac{\gamma^{2} \omega a^{-2 \gamma}}{6}\right)\right.\right.\right. \\
& \left.\left.-\frac{H}{3} \rho_{m 0} a^{\left(3 b^{2}-3+\gamma\right)}\right)-2 H^{2}\right)+\left(\left(\frac{18}{\alpha} H^{2}\left(1-\gamma-\frac{\gamma^{2} \omega a^{-2 \gamma}}{6}\right)-\frac{2}{\alpha} \rho_{m 0}\right.\right. \\
& \left.\times \quad a^{\left(3 b^{2}-3+\gamma\right)}-2 H\right)\left(\frac{6}{\alpha}\left(H^{3}\left(1-\gamma-\frac{\gamma^{2} \omega a^{-2 \gamma}}{6}\right)-\frac{H}{3} \rho_{m 0} a^{\left(3 b^{2}-3+\gamma\right)}\right)\right. \\
& \left.\left.\left.-2 H^{2}\right)+\frac{2}{\alpha} \gamma^{3} \omega a^{-2 \gamma} H^{4}-\frac{2}{\alpha}\left(3 b^{2}-3+\gamma\right) \rho_{m 0} a^{\left(3 b^{2}-3+\gamma\right)} H^{2}\right)\right) .
\end{aligned}
$$

The behavior of $\omega_{D}-\omega_{D}^{\prime}$ can be observed from Figure 4 which exhibits the freezing region.

3.5. $r-s$ plane. With the help of this plane, we can identify different DE models. Trajectories of different DE models have different ranges in this plane. For example, $\{r, s\}=\{1,0\}$ corresponds to $\Lambda C D M$ model, $\{r, s\}=\{1,1\}$ shows 


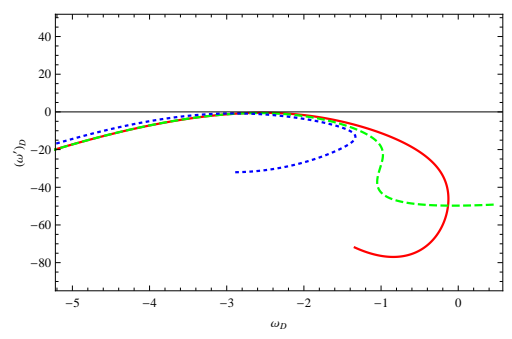

FIgURE 4. Plots of $\omega_{D}-\omega_{D}^{\prime}$ for QCD ghost DE model in fractal cosmology.

$C D M$ limit, $\{s>0, r<1\}$ shows phantom and quintessence while $\{s<0, r>1\}$ denotes chaplygin gas region. Mathematical form of state-finder parameters are given by [36]

$$
r=1+\frac{3 \dot{H}}{H^{2}}+\frac{\ddot{H}}{H^{3}} \text { and } s=\frac{r-1}{3\left(q-\frac{1}{2}\right)} .
$$

To obtain the values of $r-s$ plane we substitute Eq. (12) and (14) in (17),

$$
\begin{aligned}
r & =1+\left(\frac{6}{\alpha}\left(H^{3}\left(1-\gamma-\frac{\gamma^{2} \omega a^{-2 \gamma}}{6}\right)-\frac{H}{3} \rho_{m 0} a^{\left(3 b^{2}-3+\gamma\right)}\right)-2 H^{2}\right) \\
& \times\left(3+\frac{9 H}{\alpha}\left(1-\gamma-\frac{\gamma^{2} \omega a^{-2 \gamma}}{6}\right)-\frac{2}{H \alpha} \rho_{m_{0}} a^{\left(3 b^{2}-3+\gamma\right)}-2\right)+\frac{2 H^{4}}{\alpha} \\
& \times \gamma^{3} \omega a^{-2 \gamma}-\frac{2 H^{2}}{\alpha}\left(3 b^{2}-3+\gamma\right) \rho_{m 0} a^{\left(3 b^{2}-3+\gamma\right)} \\
s & =\frac{1}{3}\left(-\frac{3}{2}-\frac{6}{\alpha}\left(H\left(1-\gamma-\frac{\gamma^{2} \omega a^{-2 \gamma}}{6}\right)-\frac{1}{3 H} \rho_{m 0} a^{\left(3 b^{2}-3+\gamma\right)}\right)+2\right)^{-1} \\
& \times\left(\left(\frac{6}{\alpha}\left(H^{3}\left(1-\gamma-\frac{\gamma^{2} \omega a^{-2 \gamma}}{6}\right)-\frac{H}{3} \rho_{m 0} a^{\left(3 b^{2}-3+\gamma\right)}\right)-2 H^{2}\right)\right. \\
& \times\left(3+\frac{9 H}{\alpha}\left(1-\gamma-\frac{\gamma^{2} \omega a^{-2 \gamma}}{6}\right)-\frac{2}{H \alpha} \rho_{m 0} a^{\left(3 b^{2}-3+\gamma\right)}-2\right)+\frac{2 H^{4}}{\alpha} \\
& \left.\times \gamma^{3} \omega a^{-2 \gamma}-\frac{2 H^{2}}{\alpha}\left(3 b^{2}-3+\gamma\right) \rho_{m 0} a^{\left(3 b^{2}-3+\gamma\right)}\right) .
\end{aligned}
$$

The plane of this model is given in Figure 5. The $r-s$ plane for this model shows the Chaplygin gas behavior as well as $\Lambda C D M$ model.

\section{Concluding Remarks}

We have investigated the physical significance of QCD ghost DE model in fractal universe by developing various cosmological parameters as well as cosmological 


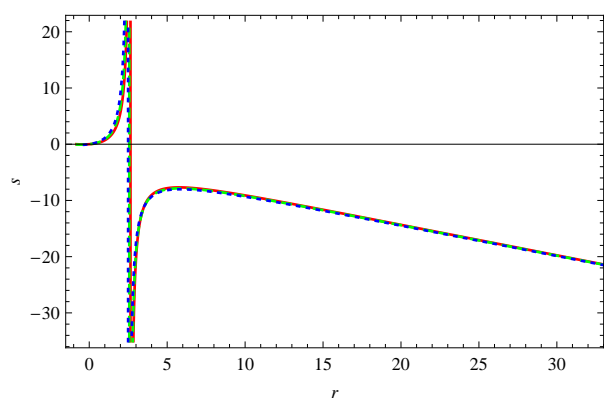

Figure 5. Plots of $r-s$ for QCD ghost DE model in fractal Cosmology.

planes. These parameters as well as planes shamefully explain the current cosmic acceleration.

\section{Competing Interests}

The author(s) do not have any competing interests in the manuscript.

\section{REFERENCES}

1. Riess, A. G. et. al. (1998). Observational evidence from supernovae for an accelerating universe and a cosmological constant. The Astronomical Journal, 116(3), 1009-1038.

2. Weinberg, S. (1989). The cosmological constant problem. Reviews of modern physics, 61(1), 1-23.

3. Kamenshchik, A., Moschella, U., \& Pasquier, V. (2001). An alternative to quintessence. Physics Letters B, 511(2), 265-268.

4. Hsu, S. D. (2004). Entropy bounds and dark energy. Physics Letters B, 594(1), 13-16.

5. Li, M. (2004). A model of holographic dark energy. Physics Letters B, 603(1), 1-5.

6. Wei, H., \& Cai, R. G. (2008). A new model of agegraphic dark energy. Physics Letters B, 660(3), 113-117.

7. Karami, K., Ghaffari, S., \& Fehri, J. (2009). Interacting polytropic gas model of phantom dark energy in non-flat universe. The European Physical Journal C-Particles and Fields, $64(1), 85-88$

8. Wei, H. (2012). Pilgrim dark energy. Classical and Quantum Gravity, 29(17), 175008.

9. Sharif, M., \& Jawad, A. (2013). Pilgrim dark energy with apparent and event horizons in non-flat universe. The European Physical Journal C, 73(10), 2600, 1-10.

10. Shamaila, S., Zafar, N., Riaz, S., Sharif, R., Nazir, J., \& Naseem, S. (2016). Gold nanoparticles: an efficient antimicrobial agent against enteric bacterial human pathogen. Nanomaterials, 6(4), 71, 2-10.

11. Amors, J., de Haro, J., \& Odintsov, S. D. (2013). Bouncing loop quantum cosmology from $F(T)$ gravity. Physical Review D, 87(10), 104037.

12. Linder, E. V. (2010). Einsteins Other Gravity and the Acceleration of the Universe. Physical Review D, 81(12), 127301.

13. Jamil, M., Momeni, D., \& Myrzakulov, R. (2013). Wormholes in a viable $F(T)$ gravity. The European Physical Journal C, 73(1), 2267.

14. Myrzakulov, R. (2012). Cosmology of $F(T)$ Gravity and k-Essence. Entropy, 14(9), 16271651. 
15. Salako, I. G., Rodrigues, M. E., \& Kpadonou, A. V. (2013). MJS Houndjo and J. Tossa. JCAP, 60, 1475.

16. Rodrigues, M. E., Salako, I. G., Houndjo, M. J. S., \& Tossa, J. (2014). Locally rotationally symmetric Bianchi type-I cosmological model in $F(T)$ gravity: from early to dark energy dominated universe. International Journal of Modern Physics D, 23(01), 1450004.

17. Baffou, E. H., Kpadonou, A. V., Rodrigues, M. E., Houndjo, M. J. S., \& Tossa, J. (2015). Cosmological viable $f(R, T)$ dark energy model: dynamics and stability. Astrophysics and Space Science, 356(1), 173-180.

18. Houndjo, M. J. S. (2012). Reconstruction of $f(R, T)$ gravity describing matter dominated and accelerated phases. International Journal of Modern Physics D, 21(01), 1250003.

19. Nojiri, S. I., \& Odintsov, S. D. (2005). Modified GaussBonnet theory as gravitational alternative for dark energy. Physics Letters B, 631(1), 1-6.

20. Bamba, K., Odintsov, S. D., Sebastiani, L., \& Zerbini, S. (2010). Finite-time future singularities in modified GaussBonnet and $F(R, G)$ gravity and singularity avoidance. The European Physical Journal C, 67(1-2), 295-310.

21. Bamba, K., Geng, C. Q., Nojiri, S. I., \& Odintsov, S. D. (2010). Equivalence of the modified gravity equation to the Clausius relation. EPL (Europhysics Letters), 89(5), 50003.

22. Rodrigues, M. E., Houndjo, M. J. S., Momeni, D., \& Myrzakulov, R. (2013). A type of LeviCivita solution in modified GaussBonnet gravity. Canadian Journal of Physics, 92(2), 173-176.

23. Houndjo, M. J. S., Rodrigues, M. E., Momeni, D., \& Myrzakulov, R. (2014). Exploring cylindrical solutions in modified $f(G)$ gravity. Canadian Journal of Physics, 92(12), 15281540.

24. Bamba, K., Capozziello, S., Nojiri, S. I., \& Odintsov, S. D. (2012). Dark energy cosmology: the equivalent description via different theoretical models and cosmography tests. Astrophysics and Space Science, 342(1), 155-228.

25. Calcagni, G. (2010). Fractal universe and quantum gravity. Physical review letters, $104(25), 251301$.

26. Calcagni, G. (2010). Quantum field theory, gravity and cosmology in a fractal universe. Journal of High Energy Physics, 2010(3), 120.

27. Elizalde, E., Nojiri, S. I., Odintsov, S. D., \& Wang, P. (2005). Dark energy: vacuum fluctuations, the effective phantom phase, and holography. Physical Review D, 71(10), 103504.

28. Yang, W. Q., Wu, Y. B., Song, L. M., Su, Y. Y., Li, J., Zhang, D. D., \& Wang, X. G. (2011). Reconstruction of new holographic scalar field models of dark energy in bransdicke universe. Modern Physics Letters A, 26(03), 191-204.

29. Granda, L. N., \& Oliveros, A. (2008). Infrared cut-off proposal for the holographic density. Physics Letters B, 669(5), 275-277.

30. Sheykhi, A. (2009). Interacting holographic dark energy in BransDicke theory. Physics Letters B, 681(3), 205-209.

31. Jamil, M., \& Sheykhi, A. (2011). Interacting entropy-corrected agegraphic-tachyon dark energy. International Journal of Theoretical Physics, 50(3), 625-636.

32. Jamil, M., Momeni, D., \& Myrzakulov, R. (2012). Stability of a non-minimally conformally coupled scalar field in $F(T)$ cosmology. The European Physical Journal C, 72(7), 2075.

33. Zhitnitsky, A. R. (2012). Contact term, its holographic description in QCD and dark energy. Physical Review D, 86(4), 045026.

34. Cai, R. G., Tuo, Z. L., Wu, Y. B., \& Zhao, Y. Y. (2012). More on QCD ghost dark energy. Physical Review D, 86(2), 023511.

35. Caldwell, R. R., \& Linder, E. V. (2005). Limits of quintessence. Physical review letters, 95(14), 141301.

36. Sahni, V., Saini, T. D., Starobinsky, A. A., \& Alam, U. (2003). Statefindera new geometrical diagnostic of dark energy. JETP Letters, 77(5), 201-206. 
Ines G. Salako

Institut de Mathématiques et de Sciences Physiques, (IMSP) 01 BP 613 Porto-Novo, Bénin and

Département de Physique - Université d'Agriculture, de Kétou BP 13 Kétou, Bénin e-mail: ines.salako@imsp-uac.org

Faiza Gulshan

Department of Mathematics, Lahore Leads, university, Lahore-54590, Pakistan

fazi.gull@yahoo.com 